\title{
Contrainte et réécriture-création dans la traduction des Exercices de style de Raymond Queneau
}

\author{
Valentina RĂDULESCU \\ Université de Craiova \\ Roumanie
}

Résumé : Cette étude propose une analyse comparée des versions en roumain (collectif de traducteurs coordonné par Romulus Bucur), en anglais (Barbara Wright) et en italien (Umberto Eco) d'une sélection de textes d'Exercices de style, de Raymond Queneau, qui présentent des degrés variables de difficultés de traduction. L'analyse entreprise essaie de confirmer l'hypothèse de recherche que nous défendons : souvent déstabilisante et difficilement surmontable, la contrainte ne devient pas pour autant une entrave à la créativité du traducteur, ni à sa fidélité envers le style de l'original ; elle est un facteur dynamisant du processus traductif, favorisant la réécriture-création.

Mots-clés : traduction, contrainte, réécriture, création, variation

\begin{abstract}
Our study presents a comparative analysis of selected texts from several translations of Queneau's Exercices de style: the Romanian version (a collective work coordinated by Romulus Bucur), the English version (Barbara Wright) and the Italian one (Umberto Eco) that illustrate the variable degrees of difficulties in translating. The analysis is meant to confirm our research hypothesis: though disruptive and often hardly surmountable, translation constraint does not stifle translator's creativity or his fidelity toward the original style; on the contrary, it stimulates the translational process and fosters the rewriting-creation.
\end{abstract}

Keywords: translation, constraint, rewriting, creation, variation

\section{Introduction}

«La pratique de la traduction [...] repose sur deux présupposés. Le premier: nous sommes tous différents. Nous parlons différentes langues, et nos visions du monde sont profondément influencées par les caractères particuliers des langues que nous parlons. Le second : nous sommes tous pareils. Nous pouvons partager, en gros et en détail, les mêmes types de sentiments, d'informations, d'accords, etc. Sans ces deux présupposés, la traduction ne pourrait pas exister.

Ni rien de ce que nous voudrions appeler la vie en société. 
La traduction est l'un des noms de la condition humaine. » (Bellos 2012, 346)

L'un de ses aspects les plus provocateurs aussi, car la traduction est constamment confrontée à un défi de taille : préserver " l'information et la valeur globale de sa source » (Bellos 2012, 343), surmonter l'ensemble de «contraintes sociales, pratiques, linguistiques ou génériques » (ibidem, 344), afin d'aboutir à un double imparfait, mais analogue de la source. Ce défi redouble en complexité lorsqu'il s'agit de traduire sous contraintes de type particulier. C'est aussi le cas d'Exercices de style de Raymond Queneau, un ensemble de 99 variations (parodiques) sur diverses figures de rhétorique (métalogismes, métaplasmes, métagraphes), sur des actes de parole, des genres du discours non littéraire ou littéraire, à partir d'un texte de base qui relate un banal " microévénement » (Pouilloux 1991, 12). Chacune de ces variations illustre une contrainte formelle différente et allie, dans une perspective ludique, construction artificielle et virtuosité linguistique. Par conséquent, pour les traducteurs de l'œuvre, « dire presque la même chose » (Eco 2003) est en égale mesure un exercice de rigueur scientifique et de jeu avec les potentialités de la langue-cible.

Cette étude propose une analyse comparée des versions en roumain (collectif de traducteurs coordonné par Romulus Bucur), en anglais (Barbara Wright) et en italien (Umberto Eco) d'une sélection de textes d'Exercices de style, qui présentent des degrés variables de difficultés de traduction. L'analyse entreprise essaie de confirmer l'hypothèse de recherche que nous défendons : souvent déstabilisante et difficilement surmontable, la contrainte ne devient pas pour autant une entrave à la créativité du traducteur, ni à sa fidélité envers le style de l'original ; elle est un facteur dynamisant du processus traductif, favorisant la réécriture-création.

\section{Exercices de style - (ré)écriture, traduction, variation}

L'idée des Exercices de Style remonte aux années '3o du siècle passé, lorsque, après avoir écouté, lors d'un concert à la Salle Pleyel, L'Art de la Fugue de Bach, Raymond Queneau et Michel Leiris évoquent l'intérêt d'une œuvre similaire sur le plan littéraire, réalisée " au moyen de variations proliférant presque à l'infini autour d'un thème assez mince » (Queneau [1963] 2014 : 2). Entre 1942 et 1945, Queneau met en œuvre ce projet : plusieurs séries d'exercices sont rédigés et publiés dans La Terre n'est pas une vallée des larmes et dans Fontaine, avant d'être réunis et publiés en volume, en 1947, aux Éditions Gallimard. En 1963 paraît l'édition illustrée Carelman- 
Massin, en 1969 paraît une édition remaniée ${ }^{1}$ chez Gallimard, suivie d'une nouvelle édition en 1973.

Notations, qui ouvre le volume, est considéré par Stefano Bartezzaghi (2014, 269) le texte qui constitue le thème des Exercices, mais il ne s'agit pas d'un texte neutre. À l'avis de Bartezzaghi (2014, 284) et de Gérard Genette (1982, 161), Récit se rapproche le plus d'un hypothétique "degré zéro » en ce qui concerne l'exposition du thème, il est écrit dans le style le plus neutre du recueil. Ce thème, soumis et ouvert à toutes les variations possibles, est devenu dans la vision de Martin Ringot un "mythe que nous pourrions appeler "l'incident de l'autobus" ou "l'homme au long cou" et dont la constante réinterprétation souligne l'universalité - et la potentialité - de l'anecdote » $(2014, \S 39)$ : le narrateur relate un incident survenu un jour quelconque, vers midi, dans un bus de la ligne $\mathrm{S}$, entre un jeune homme à long cou et à chapeau mou, dérangé par un autre voyageur qui lui marche sur les pieds à chaque arrêt. Plus tard, le narrateur aperçoit le même jeune homme en train de discuter avec un ami devant la Gare Saint-Lazare. L'ami lui conseille de mettre un bouton supplémentaire à l'échancrure de son pardessus.

Dans la préface à l'édition italienne des Exercices, Umberto Eco, note que l'effet comique et le sens de chacune des variations se définissent non seulement par rapport au texte de départ, mais aussi dans le contexte de tous les autres. Ainsi, «l'effet comique est global, il naît de l'accumulation, figure rhétorique qui domine toutes les autres et que chaque exercice contribue à exemplifier » (Eco, 2014 : XV). Bartezzaghi souligne aussi que dans l'œuvre quenienne existent en réalité deux niveaux de contrainte: «le premier niveau est constitué par la contrainte particulière qui relie chaque chapitre à son titre et ensuite la contrainte plus générale relie les quatre-vingtdix-neuf titres au titre de l'œuvre » $(2014,269)$.

On retrouve dans les Exercices les deux pôles entre lesquels oscille la création de Queneau : " d'une part, le plaisir de traiter un thème donné dans un langage inhabituel, de l'autre le plaisir d'appliquer une formule stricte sur une composition poétique » (Calvino, 2013 [1981] : 140). Le ludique et le scientifique se conjuguent pour démontrer combien la structure non pas poétique, mais narrative à l'apparence plate, mécanique, " minimaliste » des

\footnotetext{
${ }^{1}$ De la nouvelle édition de 1969, disparaissent Hai Kai, Féminin, Permutations de 2 à 5 lettres, Permutations de 9 à 12 lettres, Réactionnaire; sont ajoutés Définitionnel, Ensembliste, Lipogramme, Tanka, Translation. Plusieurs autres exercices sont maintenus, mais à titre changé : À peu près devient Homophonique, Contre-vérités - Antonymique, Homéoptotes - Homéotéleutes, Latin de cuisine Macaronique, Mathématique - Géométrique, Noble - Ampoulé, Permutations de 5 à 8 lettres - Permutations par groupes croissants de mots, Permutations de 1 à 4 mots - Permutations par groupes croissants de mots, Prétérit - Passé simple.
} 
Notations devient malléable et se «travestit » dans chaque variante réécrite par Queneau, mais aussi par chacun de ses traducteurs.

Ce que les Exercices mettent aussi en évidence c'est que dans le cas de Queneau,

«structure est synonyme de liberté : elle produit le texte et, en même temps, la possibilité de tous les autres textes virtuels qui peuvent le remplacer. C'est l'aspect novateur du concept de multiplicité «potentielle» lancé par Queneau, sous-jacent à l'idée d'une littérature née des contraintes qu'elle choisit elle-même (pour ellemême) et qu'elle applique (à elle-même). » (Calvino 2013 [1981] : 141).

À part le fait que chaque texte écrit selon des règles précises ouvre à la multiplicité potentielle des textes qui pourraient être écrits selon les mêmes règles, il ouvre aussi à la multiplicité potentielle de « toutes les lectures qui pourraient résulter de ces textes»(Calvino,1981 142). Cette idée nous semble aussi fondamentale pour le processus de traduction, car elle renvoie logiquement à la multiplicité potentielle des expériences traductives des textes sous contrainte. Par conséquent, quelle que soit la contrainte, elle ne peut que stimuler et non pas entraver le processus traductif.

Traducteur des Exercices de style en italien, Umberto Eco avoue avoir difficilement réprimé la tentation de continuer la série des variations à partir des Notations queniennes et d'actualiser d'autres possibilités ouvertes par ce texte. En ce qui le concerne, «Exercices de style est comme l'œuf de Colomb, une fois que quelqu'un a eu l'idée, il est facile de continuer ad libitum » (Eco 2014, XX). Queneau lui-même avait d'ailleurs proposé, dans l'annexe de l'édition Carelman-Massin, une liste de 124 autres exercices possibles, nouvelle provocation lancée à ses lecteurs. Eco a préféré finalement se limiter au rôle de traducteur et rester fidèle à Queneau. Mais cette fidélité allait être une particulière, car même si une partie des textes ont permis une traduction directe, la plupart ont fait l'objet d'un remaniement, d'une traduction-réécriture en langue-cible. Or il fallait trouver la méthode adéquate pour un livre d'une telle complexité. Quelle a été sa méthode (qui finalement s'est avérée la même pour les traducteurs en anglais et en roumain aussi) ? « Disons, [affirmait Eco], que Queneau a inventé un jeu et en a expliqué les règles au cours d'une partie magnifiquement jouée en 1947. Fidélité signifiait comprendre les règles du jeu, les respecter, ensuite jouer une nouvelle partie avec le même nombre de mouvements » (Eco 2014, XX). Si l'on envisage le jeu de Queneau en termes de jeux de mots, on pourrait affirmer que pour l'auteur, ainsi que pour ses traducteurs, Notations représente le ludé (Guiraud 
1979, 105) tandis que les 99 variations les ludants de Notations. Si on l'envisage en termes genettiens, les 99 textes queniens sont les hypertextes d'un hypotexte unique, des oulipismes transformationnels, qui réalisent une transtylisation (Genette 1982, 165) linguistique et formelle et actualisent de nouveaux sens « car la transformation d'un texte produit toujours un autre texte, et donc un autre sens » (ibidem, 67). La solution consistait donc à appliquer des mécanismes identiques à ceux de la création à la réécrituretraduction, afin d'obtenir des textes et des effets similaires à ceux de l'original.

Adam Thirlwell remarque, à son tour, en parlant d'Exercices de style qu'il s'agit d'un « livre d'effets linguistiques » $(2014,20)$, où, à travers le jeu des variations, une évidence s'impose: "chaque histoire est différente en fonction des mots qui la composent. La moindre modification suscite une vision nouvelle du réel » (2014, 21). Le romancier et théoricien de la traduction anglaise accorde aussi une attention particulière à l'une des questions les plus problématiques pour les traducteurs: le style. L'analyse de la traduction des Exercices de style en anglais, conduit Thirlwell à la conclusion que la traduction du style est tout à fait possible et à une définition très pertinente de ce dernier : "Barbara Wright a saisi qu'un style est un système de déformations opérées sur les phrases. Et qu'une traduction mot à mot soit parfois impossible n'empêche pas la traduction, démontrant à quel point les effets de style sont exportables ». (Thirlwell 2014, 41). Raymond Queneau appréciait d'ailleurs particulièrement la traduction anglaise de ses Exercices : « J'avais toujours pensé que rien n'est intraduisible et à présent j'en vois une nouvelle preuve ", écrivait-il à la traductrice (ibidem). Tout style, ajoute Thirlwell en élargissant sa définition, « est un système d'opérations pratiquées sur une langue dans le but de produire des effets : c'est un genre de machine. Aussi ces machines du style sontelles transportables » (Thirlwell 2014, 42). Mais afin de réussir dans cette entreprise, et dans l'aventure traductive en son ensemble, il faut d'une part, que le traducteur "se tienne légèrement à distance du texte », qu'il laisse « ses motifs et ses structures sous-jacents émerger d'eux-mêmes au cours de la réécriture dans une autre langue. » (Bellos 2012, 308) et d'autre part qu'il exploite/exerce de manière créative ce que David Bellos appelle ses pattern-matching skills :

« Le métier - skill - du traducteur consiste ainsi à trouver dans sa propre langue des correspondances relativement abstraites, susceptibles d'être réalisées en pratique entre des motifs, des figures ou des modèles aux niveaux lexical, syntaxique, contextuel, référentiel et stylistique - des patterns - présents dans la source de telle façon qu'on puisse dire : ça se marie bien. » (Bellos 2012, 309) 
On obtient de la sorte une nouvelle œuvre, substitut acceptable de l'original, mais ce que cette traduction acceptable désigne " est une relation globale entre source et cible qui n'est ni identité, ni équivalence, ni analogie, mais seulement cette chose complexe qui nous permet de reconnaître un heureux mariage. Telle est la vérité sur la traduction » (Bellos 2012, 344).

\section{Exercices de style - traduction et réécriture-création}

Comme nous l'avons mentionné précédemment, les textes des Exercices de style présentent des difficultés de traduction variables, qui oscillent entre deux pôles : celui de la traduction directe, presque littérale et celui de l'intraduisibilité : par exemple, Eco renonce à traduire Loucherbem, écrit dans un jargon trop codé, ou Homophonique, l'italien étant beaucoup plus pauvre en homophones que le français. Généralement, du côté du pôle de la traduction directe se situent des textes qui n’impliquent ni jeux de mots complexes, ni poèmes à forme fixe, ni différences grammaticales ou lexicales insurmontables entre la langue de l'original et celle du texte d'arrivée ; c'est le cas de la série d'exercices illustrant divers champs sémantiques: Visuel, Olfactif, Gustatif, Auditif, Tactile, Gastronomique, Zoologique, Botanique, de certains types de discours: Philosophique, Ampoulé, Désinvolte, Vulgaire, Rétrograde, Notations, Lettre officielle, etc., de certains genres littéraires: Comédie, Vers libres. Nous examinerons ensuite quelques exemples tirés de Récit et de Négativités ${ }^{2}$, textes susceptibles de ne pas opposer de résistance particulière à la traduction.

Récit

«Un jour, vers midi du côté du parc Monceau, sur la plateforme arrière d'un autobus à peu près complet de la ligne $S$, j'aperçus un personnage au cou fort long qui portait un feutre mou entouré d'un galon tressé au lieu de ruban. Cet individu interpelle tout à coup son voisin en prétendant qu'il faisait express de lui marcher sur les pieds chaque fois qu'il montait ou descendait des voyageurs. [...]

Deux heures plus tard, je le revis devant la gare Saint-Lazare en grande conversation avec un ami qui lui conseillait de diminuer l'échancrure de son pardessus en en faisant remonter le bouton supérieur par quelque tailleur compétent. » $(E D S F, 27)$

2 Les exemples sont tirés des éditions mentionnées dans les Références bibliographiques et seront suivis par les titres abréviés - EDSF - pour l'édition française, $E D S I$ pour l'édition italienne, EDSA pour l'édition anglaise et $E D S R$ pour l'édition roumaine - et par le numéro de page. 


\section{Svolgimento}

«Ieri la signora maestra ci ha portato a fare la consueta gita in autobus (linea S) per fare interessanti esperienze umane e capire meglio i nostri simili. Abbiamo socializzato con un signore molto buffo dal col molto lungo che portava un cappello molto strano con una cordicella attorno. Questo signore non si è comportato in modo molto educato perché a litigato con un altro signore che lo spingeva, ma poi ha avuto paura di prendersi un bel ceffone ed è andato a sedersi su un posto libero. [...]

Due ore piú tardi abbiamo incontrato lo stesso signore col collo lungo che parlava davanti a una stazione grandissima con un amico, il quale gli diceva delle cose o proposito del suo cappottino.

La signora maestro ci ha fatto asservare che questo episodio è stato molto istruttivo perché ci ha insegnato che nella vita accadono molte coincidenze curiose e che dobbiamo ossrvare con interesse le persone che incontriamo perché potremmo poi reincontrarle in altra occasione. » $(E D S I, 33)$

\section{Narrative}

" One day at about midday in the Parc Monceau district, on the back platform of a more-or-less full $\mathrm{S}$ bus (now No. 84), I observed a person with a very long neck who was wearing a soft felt hat which had a plaited cord round it instead of a ribbon. This individual suddenly addressed the man standing next to him, accusing him of purposely treading on his toes every time any passengers got on or off. [...]

Two hours later I saw him in front of the Gare Saint-Lazare engaged in earnest conversation with a friend who was advising him to reduce the space between the lapels of his overcoat by getting a competent tailor to raise the top button. » $(E D S A, 18)$

\section{Povestire}

« Într-o zi, pe la amiază, aproape de parcul Monceau, pe platforma din spate a unui autobuz aproape plin al liniei S (astăzi 84) am observat un personaj cu gâtul foarte lung care purta o pălărie de fetru foarte moale, având în jur un şnur împletit în loc de panglică. Acest individ îl interpelă deodată pe vecinul lui, pretinzând că acesta îl călca intenţionat de fiecare dată când urcau sau coborau călători. [...] Două ore mai târziu l-am revăzut în faţa gării Saint-Lazare discutând aprins cu un prieten care îl sfătuia să-şi micşoreze răscroiala pardesiului, mutându-şi puţin nasturele de sus la un croitor priceput. » $(E D S R, 44)$

La contrainte dans le cas de Récit consiste à préserver dans la langue d'arrivée la neutralité de l'original. Les traductions roumaine et anglaise l'ont fidèlement réalisée, tandis qu'Umberto Eco a opté pour une métamorphose radicale: le texte le plus neutre de 
l'ensemble est devenu l'un des plus personnels. La narration a la fraîcheur et la candeur du récit enfantin, car c'est un enfant qui relate l'incident auquel il a assisté lors d'une sortie scolaire et la morale qu'il en retient. Ainsi travesti, le texte quenien est presque méconnaissable : sa traduction n'est qu'un prétexte pour la création d'un nouvel exercice de style, qui actualise l'une des potentialités de la narration, tout en remettant subtilement en discussion la possibilité d'atteindre un degré zéro de l'écriture, ainsi que la valeur du récit neutre.

Nous considérons que Récit contient aussi une deuxième contrainte, occultée par la neutralité qui a focalisé l'attention des traducteurs : l'original contient seulement trois pauses, marquées par des virgules, bien que logiquement, le texte ait dû en contenir davantage. La segmentation atypique confère un tempo narratif particulier au texte. Tous les traducteurs du texte ont introduit une pause supplémentaire, chacun à des endroits différents du récit, mais, pour le reste, ils ont respecté la structure du texte français.

Négativités

« Ce n'était ni la veille, ni le lendemain, mais le jour même. Ce n'était ni la gare du Nord, ni la gare de Lyon mais la gare Saint-Lazare. Ce n'était ni un parent, ni un inconnu, mais un ami. Ce n'était ni une injure, ni une moquerie, mais un conseil vestimentaire. » $(E D S F, 29)$

Negatività

« Non ieri, non domani, il giorno stesso. Né alla Gare du Nord ne alla Gare de Lyon: la Gare era Saint-Lazare. Non era con parenti o con serpenti, ma con uno dei suoi conoscenti. Che non l'insultava né lo lodava ma gli suggeriva - circa il cappotto che portava. » $(E D S I, 37)$

\section{Negativities}

«It was neither the day before nor the day after, but the same day. It was neither the Gare du Nord nor the Gare du P.-L.-M., but the Gare Saint-Lazare. It was neither a relation nor a stranger, but a friend. It was neither insult nor ridicule, but sartorial advice. » $(E D S A, 20)$

\section{Negativități}

« Nu era nici în ajun, nici a doua zi, ci chiar în acea zi. Nu era nici Gara de Nord, nici Gara Lyon, ci Gara Saint-Lazare. Nu era nici o rudă, nici un necunoscut, ci un prieten. Nu era nici insultă, nici batjocură, ci un sfat vestimentar. » $(E D S R, 46)$

La contrainte imposée par Négativités permet également une traduction directe. Chaque phrase a une structure similaire: une négation répétée, suivie d'une oppositive, structure facilement reproductible dans les trois langues-cible. On remarque la fidélité des trois versions envers le texte-source, mais un léger écart est 
enregistré dans la traduction italienne : si dans la première partie du texte Eco reste fidèle à l'original, dans la dernière partie il opte pour une structure plus elliptique: le verbe est supprimé, procédé qui accélère le rythme du texte et en accentue aussi la structure « hachée ». En outre, le jeu de rimes et l'allitération des deux dernières phrases soulignent la dimension ludique de la version italienne.

Plus près du pôle opposé, les poèmes à forme fixe et les jeux de mots sont un défi pour tout traducteur et leur traduction directe est rarement possible. Dans ce cas, c'est le principe d'équivalence qui devient opérant et les traducteurs aboutissent souvent à des traductions adaptatives ou à la recréation des textes dans la langue d'arrivée; leur créativité et leurs pattern-matching-skills sont fortement sollicités. Si nous avons toujours soutenu - et c'est notre conviction intime - que dans tout texte littéraire traduit on entend conjointement la voix de l'auteur et celle du traducteur, à intensité égale, il nous semble que dans la traduction des poèmes à forme fixe et des jeux des mots la voix de l'auteur ne fait qu'accompagner en sourdine celle du traducteur. Et même si le résultat du processus traductif est un double plus ou moins méconnaissable de l'original, il faut toujours garder en mémoire que «l'une des vérités de la traduction - l'une des vérités que la traduction enseigne - est que tout est dicible. Surtout la poésie. [...] La poésie n'est pas ce qui se perd [dans le travail des traducteurs], elle est ce qui s'y gagne » (Bellos 2012, 166). C'est une renaissance sous une autre apparence, mais qui en garde l'essence.

Dans cette perspective, les versions italienne, anglaise et roumaine de Sonnet font entendre les voix distinctes de leurs traducteurs et proposent, de même que l'original, une réécriture parodique du sonnet classique ou romantique :

Sonnet

"Glabre de la vaisselle et tressé du bonnet, Un paltoquet chétif au cou mélancolique

Et long se préparait, quotidienne colique,

À prendre un autobus le plus souvent complet. » $(E D S F, 85)$

\section{Sonetto}

« Tanto gentile la vettura pare

Che va da Controscarpa a Ciamperetto

Che le genti gioiose a si pigiare

Vi van, e va con esse un giovinetto. » $(E D S I, 123)$

Sonnet

"Glabrous of dial, a plait upon his bonnet, This lousy lout - how sad the neck he bore 
And long also) - performed his usual chore:

Tha bus was full and he tried to get on it. » $(E D S A, 65)$

\author{
Sonet \\ « Cu şnur la pălărie, burlanul spân şi fin, \\ Un individ plăpând, un gât lung trist ridică, \\ Se pregăteşte, iată, de zilnica colică \\ Să prindă'autobuzul, cel mai adesea plin. » $(E D S R, 90)$
}

La contrainte formelle est respectée, mais des différences apparaissent entre le contenu et les effets de chaque texte : l'onde de mélancolie, de tristesse, ainsi que l'apparence chétive du jeune homme, se retrouvent dans les versions anglaise et roumaine, tandis que Umberto Eco fait une fois de plus exception, car il introduit une note de légèreté et de joie: sa version acquiert ainsi une double visée: parodier le sonnet, en général, et le texte quenien en particulier. Du point de vue du contenu et du style, la version roumaine est la plus proche de l'original, mais finalement, ce qui importe dans la traduction de Sonnet n'est pas le contenu, mais plutôt le plaisir du jeu d'effets textuels.

Le célèbre lipogramme transformationnel (absent de la version anglaise du recueil) montre que la traduction des jeux des mots repose encore plus sur l'exploitation des mécanismes de création de l'original et que les potentialités multiples du texte qu'elle actualise sont engendrées par la contrainte-même. Queneau a écrit un lipogramme en $e$ et tous les traducteurs ont retravaillé le contenu du texte pour arriver à des versions équivalentes, sans employer la voyelle $e$. Eco, fidèle à son rôle de traducteur, mais encore plus à celui d'écrivain et de sémioticien n'a pas résisté à la tentation de créer plusieurs variantes en italien, en respectant contrainte générale du lipogramme (l'absence de l'une ou de plusieurs lettres de l'alphabet). Il a créé, de la sorte, cinq versions lipogrammatiques (en $a, e, i, o, u$ ), dont le ton varie entre le familier cocasse dans Lipogramma in o et la neutralité dans Lipogramma in u. La version roumaine privilégie un mélange de roumain standard et de jargon modéré, le changement rapide de style dans le texte très bref créant un effet comique, mais aussi un nouveau centre d'intérêt pour le lecteur. Mais, Lipogramme semble un texte-piège, qui ne livre pas facilement tous ses secrets, où une contrainte en cache une autre, du même type: dans "Archéologie de l'Oulipo en Exercices chez Queneau pour une approche communicationnelle du fait littéraire », Emmanuel Souchier a démontré que le texte quenien (qui contient un et perturbateur) est également un lipogramme en quatre autres lettres : $j, k, w$ et $z$ (cf. Ringot 2014, § 32). Une contrainte peu évidente, donc, pour les lecteurs et pour les traducteurs: Eco, de même que les 
traducteurs roumains, ont fait fausse route. Mais, par un pur hasard (ou peut-être pas ?) linguistique, Lipogramma in $i$ actualise aussi la deuxième contrainte. Cela prouve que, quelque travaillée qu'elle soit, la traduction est parfois in-formée par les fulgurations du hasard, dont les «offres » sont consciemment ou inconsciemment assimilées, exploitées par les traducteurs.

\section{Lipogramme}

«Voici.

Au stop, l'autobus stoppa. Y monta un zazou au cou trop long, qui avait sur son caillou un galurin au ruban mou. Il s'attaqua aux panards d'un quidam dont arpions, cors, durillons sont avachis d'un coup; puis il bondit sur un banc et s'assoit sur un strapontin où nul n’y figurait. » $(E D S F, 111)$

\section{Lipogramma in o}

« Un bel dí, alle undici piu che passate, diversamente che sul davanti di una vettura della linea $S$, guarda guarda un gagà, quasi una giraffa, che a sulla testa una faccenda tutta intrecciata. Ululante, il cretinetti dice a un passeggiere che gli pesta i piedi a ciascuna fermata. Ma repetinamente smette e va a cadere su un sedile che sta più in là, senza che altri vi sieda. » $(E D S I, 162)$

\section{Lipogramma in $\mathrm{u}$}

«Era mezzogiorno, e sopra la piattaforma posteriore del veicolo collettivo di linea S vedo il giovinotto: collo non certo corto cappello con cordicella intrecciata. Egli apostrofa il vicino dicendo che gli pesta i piedi ad ogni fermata et ad ogni discesa di passeggero. Poi si calma, tace, e va a prendere il posto che si è appena liberato. » $(E D S I, 162)$

\section{Lipogramă}

« Iată.

La stop autobuzul a stopat. A urcat un ciudat cu gâtul cam lung, purtând în cap o pălăriuță cu șnur mătăsos. S-a poticnit în cracii unui gagiu a cărui călcătură, tropăială și bătătură s-au strâmbat dintrodată; apoi a sărit asupra unui loc și a ocupat o strapontină goală. » $(E D S R, 108)$

Le savoureux Poor lay Zanglay, illustre brillamment l'alliance de la créativité de l'écrivain et du traducteur et le "principe machinal » auquel ils s'en remettent " pour tirer de leur hypotexte (baptisé par Perec "texte-souche") un texte lexicalement tout différent » (Genette 1982, 63). Queneau parodie les guides de conversation pour les étrangers dans un exercice de graphie pseudophonétique hilarant. D'une part, les exercices de virtuosité linguistique, que la «transformation ludique » (Genette 1982, 73) de l'original a exigés, confirment la théorie de Thirlwell, les versions 
anglaise, roumaine et italienne étant des exemples de remaniement du texte très fidèles au style de Queneau et aux effets textuels de l'original. D'autre part, le texte étant pratiquement intraduisible, la seule option viable pour les traducteurs s'est avérée celle théorisée par Umberto Eco - jouer le même jeu que celui de l'auteur, appliquer les mêmes mécanismes de création, adapter la graphie de chaque mot de la langue-cible à la phonétique anglaise, ou, dans les cas de Barbra Wright, qui a eu la tâche la plus difficile, adapter la graphie de l'anglais à la phonétique française.

Queneau s'avère dans ce cas un traducteur au même titre que ses traducteurs, tandis que ces derniers recréent dans leurs langues respectives de nouveaux « originaux ». Les traductions présentent des variations multiples par rapport à l'original, mais on remarque également l'identité entre le choix de Queneau et ceux de ses traducteurs quant à la graphie de certains sons de l'anglais, tels [u] et [i] rendus dans les textes par oo respectivement ee :

«Ung joor vare meedee ger preelotobüs poor la port Changparay; Oon jornow versaw matzodjornow soola peattaphormah pawstareoaray dee oon howtoboos da li leenea S veedee oon johvanay [...]; Intr-o zee spre amiazer am looat otobiusul spre Poarta Champerret. Era aprowape pleen. »

La cohérence, ainsi que l'homogénéité et la fluidité de chaque texte sont aussi évidentes.

Poor lay Zanglay

"Ung joor vare meedee ger preelotobüs poor la port Changparay. Eel aytay congplay, praysk. Jer mongtay kang maym ay lar jer vee ung ohm ahveyl ung long coo ay ung chahrpo hangtooray dünn saughrt der feessel trayssay. Sir mirssyer sir mee ang cauhlayr contrer ung ingdeeveedüh kee lühee marshay sühr lay peehay, pühee eel arlah sarsswar.

Ung per plüh tarh jer ler rervee dervang lar Garsinglahzahr ang congparhrgnee d'ung dangdee kee lühee congsayhiay der fare rermongtay d'ung cran ler bootong der song pahrdessüh. »(EDSF, 129)

Perlee Englaysee

"Oon jornow versaw matzodjornow soola peattaphormah pawstareoaray dee oon howtoboos da li leenea S veedee oon johvanay dull calloh trop-o-loongo key portavah oon cappellow cheercondutaw di oona cordichalla intretch-chce-ah-tah. Hesso apostraphaw eel soooh veeceenaw deeschandaw key phacheeavah hap-postah ha pestarlee ee peadee toota la volta key kwalkoonaw sallevah o'smontavah.

Inffenay abbandonaw lah discussionay par jettarsee soo de oon postaw lebaraw. Law reveedee tampo dohpoh hallah Garsintlahzahr 
caw noon companeo key lee sujehrreevah dee faray reesaleera oon poh-ooh eel buttone superioray da eel soprabeetaw. » $(E D S I, 191)$

For ze Freinetche

« Oine deille abahoute middeille, Aï gotte inetou a basse ouitche oise goïngue in zi directionne ave zi Porte Champerret. Ite oise foule, nirlie. Aï gotte ine aule ze séïme ainede. Aï seau a manne ine ite hou hade a longue necque ainede a hatte ouise a sorte aove platède corde rounde ite. Zisse manne gotte aingrie ouise a tchappe hou oise traidingue onne hise tose, ainede zène ouènete and satte dahoune.

A bitte leïter Aï seau againe ine fronte ove ze Gare Saint-Lazare ouise a danedie hou oise advaïzingue hime tou mouve hise oveurcote batonne a litteule bitte ayère eup. » (EDSA, 101).

\section{Pentroo engleji}

«Intr-o zee spre amiazer am looat otobiusul spre Poarta Champerret. Era aprowape pleen. Totushi am urcat shi am verzut oon berbat $\mathrm{cu}$ geetool long shi o palerye inconjoorater cu oon fel de sfoare împletiter. Atchest domn se enerve pe oon indeeveed kare eel kalca pe pitchiwoare, apoi se doose sa se asheze.

Putsin my tirzeew l-am reverzut in fatsa Gaari Saint-Lazare in company a oonooi dandy kare il sfertooia sa-shi moote putsin nastoorele pardesiewului. » $(E D S R, 123)$

\section{Conclusion}

Dans Exercices de style, la réécriture du thème dans les textes de l'original et dans leurs versions italienne, anglaise et roumaine est basée sur quatre aspects: ludique, transformationnel, machinal, créatif. En ce qui concerne la traduction, ces quatre aspects se retrouvent autant dans les textes issus d'une traduction directe, que dans ceux qui ont été plus ou moins remaniés dans les langues-cible, suite à des difficultés de traduction majeures ou à la volonté du traducteur. Tout comme l'original, les versions en italien, en anglais et en roumain exhibent les potentialités formelles, sémantiques et stylistiques de chaque texte, ainsi que les potentialités créatives des traducteurs, qui s'actualisent dans la traduction/l'écriture sous contrainte.

Les trois traductions sont stylistiquement très fidèles à l'original, malgré certains écarts et certains remaniements inévitables dans la traduction-recréation et illustrent le concept de multiplicité potentielle. Il faut aussi noter que les trois traductions sont, pour reprendre la formule d'Adam Thirlwell, des livres «d'effets linguistiques » très aboutis, qui n'ont pas pris une ride dans le temps. Par ailleurs, en nous rapportant cette fois aux paroles de Martin Ringot, qui affirme que "d'un unique texte, il peut exister une multitude de traductions possibles dans la mesure où la traduction, 
la version fidèle en tous points ne peut pas exister » (2014, § 10), soulignons le caractère ouvert de l'original et de ses traductions : un original qui pourrait être à tout moment enrichi par de nouveaux exercices, en commençant par ceux contenus dans la liste de Queneau lui-même, et des traductions à tout moment perfectibles. Exercices de style est pour nous le livre-réseau par excellence, exemple d'hypertexte palimpseste des hypertextes contemporains, qui continue à se métamorphoser grâce à sa traduction dans de nouvelles langues et qui pourrait proliférer incessamment, grâce à la réécriture du thème et à sa traduction/retraduction continuée.

\section{Références bibliographiques}

BELLOS, David. Le poisson et le bananier. Une histoire fabuleuse de la traduction (traduction de l'anglais par Daniel Loayza avec la collaboration de l'auteur). Paris: Flammarion, 2012 [Is That a Fish in Your Ear? Translation and the Meaning of Everything, Penguin Books Ltd., 2011].

BARTEZZAGHI, Stefano. "Comme si diventa scrittoranti. Effetti e transizioni negli Esercizi di stile ». Dans Raymond Queneau, Esercizi di stile (Introduzione e traduzione di Umberto Eco. Nuova edizione a curà di Stefano Bartezzaghi). Torino : Einaudi, 2014.

CALVINO, Italo. "The Philosophy of Raymond Queneau ». Dans Raymond Queneau, Exercises in style (translated par Barbara Wright, illustrations by Stefan Themerson). Richmond: Alma Classics, 2013 [1981].

ECO, Umberto. Dire Presque la même chose. Expériences de traduction (traduit de l'italien par Myriem Bouzaher). Paris : Grasset, 2006 [Dire quasi la stessa cosa. Esperienze di traduzione. Milan : Bompiani, 2003].

ECO, Umberto. «Introduzione ». Dans Raymond Queneau, Esercizi di stile (Introduzione e traduzione di Umberto Eco. Nuova edizione a curà di Stefano Bartezzaghi). Torino : Einaudi, 2014.

GENETTE, Gérard. Palimpsestes : La littérature au second degré. Paris : Éditions du Seuil, collection « Points-Essais », 1982.

GUIRAUD, Pierre. Les Jeux de mots. Paris : P.U.F., 1979 [1976].

POUILLOUX, Jean-Yves. Les Fleurs bleues de Raymond Queneau. Paris : Gallimard, collection Folio, 1991.

RINGOT, Martin. "Traduction sous contrainte d'un livre mythique ". Cahiers d'études romanes [en ligne], $\mathrm{n}^{\circ} 29$ | 2014. Mis en ligne : le 6 avril. URL : http://etudesromanes.revues.org/4667. 10.4000/etudesromanes.4667 [consulté le 29 août 2016].

SOUCHIER, Emmanuel. "Archéologie de l'Oulipo en Exercices chez Queneau pour une approche communicationnelle du fait littéraire ». $L a$ Licorne, $\mathrm{n}^{\circ}$ 100. 50 ans d'Oulipo : de la contrainte à l'œuvre. Rennes : Presses Universitaires de Rennes, 2012.

THIRLWELL, Adam. Le Livre multiple (traduction de l'anglais par AnneLaure Tissut). Paris : Éditions de L'Olivier, 2014. 


\section{Corpus}

QUENEAU, Raymond.

Exercices de style. Paris: Gallimard, collection «Folio », 2016

[1947, 1973].

Esercizi di stile (Introduzione e traduzione di Umberto Eco. Nuova edizione a curà di Stefano Bartezzaghi e l'introduzione, scritta da Queneau per un'edizione del 1963). Torino : Einaudi, 2014 [1983, 2001, 2008].

Exercises in style (translated par Barbara Wright, illustrations by Stefan Themerson). Richmond: Alma Classics, 2013 [première publication en anglais par Gaberbocchus en 1958].

Exerciţii de stil (traducere în limba română de Romulus Bucur ; Luminiţa Boază, Irina Grădinariu, prefaţă de Luca Piţu). Piteşti : Editura Paralela 45, 2013. 